\title{
Fatores de Risco Associados à Mortalidade em Pacientes com Sepse em Unidade de Terapia Intensiva de Hospital Privado de Pernambuco*
}

\author{
Risk Factors Associated to Mortality on Septic Patients in an \\ Intensive Care Unit of a General Private Hospital from Pernambuco
}

Joana Corrêa de A. Koury ${ }^{1}$, Heloísa Ramos Lacerda ${ }^{2}$, Alberto José de Barros Neto ${ }^{3}$

\section{RESUMO}

JUSTIFICATIVA E OBJETIVOS: Verificar a associação entre as características clínicas, epidemiológicas e laboratoriais com a mortalidade de pacientes com sepse, internados em UTI de hospital privado do estado de Pernambuco (Nordeste do Brasil), a fim de melhorar o atendimento a essa população, através da identificação precoce dos pacientes com risco de desenvolver falência de órgãos.

MÉTODO: Estudo de caso-controle aninhado a uma coorte prospectiva e observacional que incluiu os pacientes adultos admitidos na UTI com sepse ou que a desenvolveram durante a internação. Foram colhidos os dados epidemiológicos, avaliados os escores clínicos e exames laboratoriais como: D-dímero, antitrombina III, INR, contagem de plaquetas, sódio, albumina,

1. Mestre em Medicina Tropical/ UFPE, Hematologista da Fundação HEMOPE, Recife-PE, Médica da UTI do Real Hospital Português.

2. Professora Doutora Adjunta do Departamento de Medicina Clínica, CCS - UFPE e da UPE, Coordenadora do Programa de Pós-Graduação em Medicina Tropical, Vice-Coordenadora da UTI do Hospital Esperança.

3. Presidente da Sociedade de Terapia Intensiva de Pernambuco (SOTIPE), Chefe da UTI do Real Hospital Português, Especialista em Terapia Intensiva pela Associação de Medicina Intensiva Brasileira (AMIB)

${ }^{*}$ Recebido do Real Hospital Português, Recife, PE

Apresentado em 11 de setembro de 2006

Aceito para publicação em 15 de janeiro de 2007

Endereço para correspondência:

Dra. Joana Corrêa de A. Koury

Real Hospital Português, UTI Geral $1{ }^{\circ} \mathrm{A}$

Av. Gov. Agamenon Magalhães,4760, Paissandú

52010-900 Recife, PE

Fone: (81) 3416-1053

E-mail: joanakoury@ig.com.br

(C)Associação de Medicina Intensiva Brasileira, 2007 lactato e creatinina, sendo analisada sua associação com a mortalidade. Os pacientes foram acompanhados até a alta da UTI ou óbito.

RESULTADOS: Foram incluídos no estudo 199 pacientes. Após regressão logística, apenas o tempo de internação na UTI maior que $72 \mathrm{~h}$, a presença de doença crônica associada, o número de órgãos acometidos superior a três e o lactato maior que $4 \mathrm{mmol} / \mathrm{L}$ estiveram associados com a mortalidade. Com relação à associação com o intervalo de tempo para o óbito, apenas o escore SOFA foi significativo, pois um terço dos pacientes com pontuação superior a 12 foram a óbito em menos de $72 \mathrm{~h}$.

CONCLUSÕES: Os pacientes admitidos com sepse na UTI provenientes da comunidade (tempo de internação hospitalar $<72$ horas) apresentaram melhor prognóstico, os pacientes com maior número de órgãos com disfunção apresentaram risco de óbito mais elevado. Laboratorialmente apenas o lactato elevado esteve associado com a mortalidade.

Unitermos: epidemiologia, falência de órgãos, prognóstico, sepse.

\section{SUMMARY}

BACKGROUND AND OBJECTIVES: Verify the association between clinical, epidemiological and laboratorial characteristics with mortality of septic patient in an Intensive Care Unit (ICU) from Pernambuco, northeast of Brazil, to improve the attention for patients with sepse which are in risk of developing organ dysfunction.

METHODS: Case-control study, without intervention, that included adults' patients admitted in ICU with sepsis or that developed it during ICU stay.

RESULTS: It was included 199 patients. After logistic regression, the length of hospital stay more than 72 hours before admission in ICU, evidence of associa- 
ted co-morbidities, more than three organ failures, and lactate more than $4 \mathrm{mmol} / \mathrm{L}$ were associated with mortality. The SOFA score with more than 12 points was associated with precocity mortality $(<72$ hours).

CONCLUSIONS: The septic patients admitted ICU with less than $72 \mathrm{~h}$ of hospital stay have a better prognosis, and those with a great number of organ failure, and co-morbidities have a superior mortality rate. Between laboratory results, only the high concentration of lactate is associated with mortality.

Key Words: epidemiology, organ failure, prognosis, sepsis

\section{INTRODUÇÃO}

A sepse é uma síndrome clínica de resposta inflamatória sistêmica secundária a um processo infeccioso com foco presumido ou conhecido ${ }^{1,2}$. No Brazilian Sepsis Epidemiological Study (BASES) ${ }^{3}$, realizado em UTI do sudeste e sul do país, a incidência de sepse e sepse grave foi de 61,4 e 35,6 por 1000 pacientes-dia, respectivamente. Angus e col., realizaram um estudo epidemiológico em 2001, para analisar a incidência e prognósticos dos pacientes com sepse grave ${ }^{4}$. Nessa pesquisa foi estimada a incidência de sepse nos Estados Unidos em 751.000 casos por ano ( 3 casos por 1000 indivíduos e 2,26 casos por 100 altas hospitalares), com mortalidade de $28,6 \%$, ou 215.000 óbitos em todo país. Observou-se, também, que tanto a incidência quanto a mortalidade por sepse grave aumentaram com a idade. Um estudo semelhante, realizado na Austrália e Nova Zelândia, mostrou uma incidência de 0,77 por 1000 indivíduos com mortalidade de $26,5 \%{ }^{5}$.

Na sepse os tecidos distantes do foco inicial da infecção apresentam sinais de inflamação, que incluem vasodilatação, aumento da permeabilidade microvascular e acúmulo de leucócitos ${ }^{5}$. A sepse pode ser autolimitada ou progredir para a sepse grave e choque séptico, onde as anormalidades circulatórias (depleção do volume intravascular, vasodilatação periférica, depressão miocárdica e aumento do metabolismo), levam ao desequilíbrio entre a necessidade e a demanda de oxigênio, resultando em hipóxia global ou choque. A hipóxia tecidual reflete a gravidade da doença e é preditiva do desenvolvimento da disfunção de múltiplos órgãos ${ }^{1,3,6}$.

A sepse grave é decorrente de resposta inflamatória generalizada e pró-coagulante a uma infecção. O resultado final pode ser a lesão endovascular difusa, o comprometimento de múltiplos órgãos e o óbito ${ }^{7,8}$. A disfunção múltipla de órgãos e sistemas (DMOS) está relacionada com a presença de disfunção de órgãos, em paciente com doença aguda, de forma que a homeostase não pode ser mantida sem intervenção; é considerado um processo resultante da resposta inflamatória sistêmica ao trauma, a isquemia ou a processo infeccioso $0^{9,10}$.

A DMOS é a principal causa de morte nas UTI, afetando principalmente os pacientes sépticos. Os seus fatores de risco são idade, doença crônica prévia, reanimação inadequada, foco inflamatório e infeccioso persistente ${ }^{2}$. A falência de órgãos tem efeito aditivo na mortalidade, aonde $70 \%$ dos pacientes com três ou mais falências vão a óbito ${ }^{11}$.

A avaliação da qualidade e da intensidade da resposta inflamatória pode levar a identificação de pacientes em risco de disfunção de órgãos ${ }^{6}$. Um bom indicador para sepse deve fazer mais do que prever sobrevida dos pacientes, deve guiar o tratamento e revelar a sua respos$\mathrm{ta}^{12}$. Não existe um indicador isolado capaz de prover esta informação, o mais provável é que seja necessário um conjunto de indicadores de inflamação e imunossupressão ${ }^{6}$. Vários escores prognósticos que associam diferentes parâmetros clínicos e laboratoriais como o Acute Physiology and Chronic Health Evaluation (APACHE II) e o Sepsis Related Organ Failure Assessment (SOFA) são utilizados para avaliar o risco de morte ${ }^{13,14}$. Entretanto, sabendo-se da participação do endotélio e, particularmente, do sistema de coagulação na sepse, tenta-se avaliar se algum dos novos parâmetros utilizados na prática clínica, como o D-dímero, a antitrombina III (AT), o International Normatizated Ratio (INR), ou mesmo os exames mais tradicionais como o lactato, a contagem de plaquetas e o fibrinogênio podem predizer isoladamente o risco de morte ${ }^{15-19}$. A importância dos sistemas de coagulação e fibrinólise no desenvolvimento da DMOS têm sido estudadas por vários autores. Já foi demonstrado que o complexo trombina-antitrombina foram maiores nos pacientes sépticos que desenvolveram falência de órgãos ${ }^{9}$, como também foi observado que o escore SOFA estava mais elevado nos pacientes com coagulação intravascular disseminada e que os níveis de AT reduzidos se correlacionavam com maior disfunção de órgãos e pior prognóstico?. O objetivo desse estudo foi verificar a associação entre os diferentes parâmetros clínicos, epidemiológicos e laboratoriais com a mortalidade de pacientes com sepse internados numa UTI privada do estado de Pernambu$\mathrm{co}$, bem como verificar a associação entre distúrbios de coagulação (plaquetopenia e redução da atividade da antitrombina) com a disfunção de múltiplos órgãos. 


\section{MÉTODO}

A pesquisa foi aprovada pela Comissão de Ética do Centro de Ciências da Saúde da Universidade Federal de Pernambuco (protocolo $N^{\circ} 187 / 2004$ ), uma vez que o hospital não possui Comitê de Ética em Pesquisa. Os pacientes ou seus familiares assinaram um termo de consentimento informado.

O trabalho foi realizado na UTI geral do Real Hospital Português de Beneficência situado na cidade de Recife-PE, Brasil. Trata-se de hospital privado de cuidados terciários com 525 leitos e uma UTI geral composta de 26 leitos. O hospital possui uma UTI cardiológica e uma UTI neurológica que não foram incluídas no estudo.

O desenho do estudo foi do tipo caso-controle aninhado a uma coorte prospectiva e observacional, onde foram incluídos, de forma consecutiva, todos os pacientes adultos que apresentavam sepse no momento da internação na UTI ou desenvolvida após a admissão, no período de agosto a dezembro de 2004. A amostra foi não aleatória e o seu tamanho foi calculado no Epi-info $6.0 \mathrm{com}$ valor de beta $=0,2$ e alfa $=0,05$ para um estudo do tipo caso-controle, tendo sido estimado o tamanho mínimo da amostra para cada variável independente usada no estudo. O grupo de casos foi definido como o de pacientes sépticos que evoluíram para o óbito e o grupo-controle como pacientes sépticos que receberam alta da UTI, não houve pareamento por idade ou sexo.

A sepse foi definida como um processo infeccioso associado a dois ou mais critérios da síndrome da resposta inflamatória sistêmica: temperatura $\geq 38^{\circ} \mathrm{C}$ ou $\leq 36^{\circ} \mathrm{C}$; freqüência cardíaca $\geq 90 \mathrm{bpm}$; freqüência respiratória $\geq$ $20 \mathrm{rpm}$, ou $\mathrm{PaCO}_{2} \leq 32 \mathrm{mmHg}$, ou necessidade de ventilação mecânica; leucócitos $\geq 12.000$ células $/ \mathrm{mm}^{3}$, ou $<4.000$ células $/ \mathrm{mm}^{3}$, ou $10 \%$ de células imaturas; os pacientes com sepse grave além dos critérios descritos apresentavam ao menos uma disfunção orgânica e no choque séptico, além de preencherem os critérios de sepse, apresentavam pressão arterial sistólica $\leq 90$ $\mathrm{mmHg}$ após reposição volêmica com necessidade de fármacos vasoativos ${ }^{21}$. Foram excluídos do estudo os pacientes que retornaram para a UTI, com novo episódio de sepse, após já terem participado do estudo na internação anterior.

A sepse foi considerada comunitária nos pacientes com menos de $72 \mathrm{~h}$ de internação hospitalar prévia à admissão na UTI e nosocomial quando o tempo de internação foi maior que $72 \mathrm{~h}$.

Todos os pacientes admitidos na UTI tiveram o esco- re APACHE II calculado nas primeiras $24 \mathrm{~h}$ da admissão. Após o diagnóstico de sepse os pacientes foram avaliados por três dias consecutivos para coleta de dados e reavaliados ao fim da internação na UTI para definição do desfecho clínico: alta ou óbito na UTI. A coleta de dados clínicos e laboratoriais foi realizada por um dos pesquisadores através de exame do paciente e análise da história clínica registrada em prontuário. Os testes laboratoriais foram feitos em um único laboratório.

Para o escore APACHE II foram utilizados os piores dados clínicos e laboratoriais das primeiras 24 horas da internação na UTI. As informações referentes ao SOFA foram obtidas por três dias a partir do dia em que o paciente foi incluído no estudo. Os critérios utilizados para avaliar a disfunção de órgãos foram os do escore SOFA. Os exames de coagulação, albumina sérica foram colhidos até $48 \mathrm{~h}$ após a inclusão no estudo. A dosagem do lactato não foi seriada, sendo utilizada a primeira dosagem realizada até $48 \mathrm{~h}$ após o diagnóstico de sepse para análise. Com relação aos exames microbiológicos, foram registrados os resultados relativos às primeiras culturas coletadas após o diagnóstico da sepse. Tendo sido colhidas duas amostras de hemoculturas para pesquisa de bactérias aeróbicas, anaeróbicas e fungos.

A presença de comorbidades, definida como qualquer doença crônica prévia à admissão na UTI, documentada em prontuário, também foi avaliada como fator prognóstico 22 .

Ao final foi avaliada a associação entre as variáveis independentes e o risco de óbito. A análise foi realizada com auxílio do programa Statistical Package for Social Sciences (SPSS) Inc./9.0 para Windows. Foi utilizado o teste de Qui-quadrado de Pearson para associação das variáveis categóricas, sendo considerado estatisticamente significativo o valor de $p<0,05$; bem como foi calculado o odds ratio (OR) para estimativa de risco e o intervalo de confiança de $95 \%$. Para a análise de regressão logística entraram todas as variáveis com $p$ $<0,200$ (tempo de internação, presença de comorbidades, ratio TTPA $>1,5$, INR $>1,5$, plaquetas $<100.000$, antitrombina $<70 \%$, lactato $>4 \mathrm{mmol} / \mathrm{L}$, albumina $<$ $2 \mathrm{~g} / \mathrm{dL}$, pressão arterial média $<70 \mathrm{mmHg}$, número de órgãos com disfunção > 3), a fim de neutralizar os efeitos das variáveis de confusão. Essa análise foi feita por blocos (um com dados clínicos e outro com dados laboratoriais) e, os que permaneceram com $p<0,05$, nesta primeira etapa, foram para o modelo de regressão logística final. Foi retirado da análise multivariada 
os escores SOFA e APACHE II por que já são fatores prognósticos devidamente consagrados.

\section{RESULTADOS}

A amostra foi composta por 199 pacientes, sendo $56,3 \%$ do sexo masculino, e $67,8 \%$ com mais de 65 anos. A média do APACHE II foi de 18,2 $\pm 6,3$ e a média do SOFA no primeiro dia de diagnóstico da sepse foi de $6,3 \pm 3,7$. A maior parte da população estudada apresentava sepse grave (73,4\%). Cerca de $43,7 \%$ dos pacientes evoluíram para óbito e 56,3\% tiveram alta da UTI. Demais dados epidemiológicos da população estudada foram descritos em estudo prévio ${ }^{22}$.

Não houve associação significativa entre o desfecho para o óbito e o sexo ou a faixa etária dos pacientes
(Tabela 1). O tempo de internação no hospital superior a $72 \mathrm{~h}$ antes da internação na UTI esteve associado com risco 1,88 maior de óbito do que menor tempo de hospitalização. A presença de doença crônica associada elevou o risco de óbito em 2,05 vezes. Entretanto o diagnóstico de doença clínica aguda, politraumatismo ou doença cirúrgica à admissão na UTI não teve associação significativa com o óbito (Tabela 2).

A presença da sepse no momento de admissão na UTI ou o desenvolvimento de sepse após a admissão não mostrou associação com o risco de morte, o mesmo ocorrendo em relação ao órgão de origem da sepse e o isolamento do agente etiológico (Tabela 3).

Os pacientes com o INR > 1,5, contagem de plaquetas inferior a $100.000 / \mathrm{mm}^{3}$ e atividade da AT menor que $70 \%$ apresentaram maior risco de óbito com OR

Tabela 1 - Associação das Variáveis Sexo e Faixa Etária segundo a Evolução dos Pacientes Admitidos na UTI.

\begin{tabular}{|c|c|c|c|c|c|c|c|}
\hline \multirow{3}{*}{ Variáveis } & \multicolumn{4}{|c|}{ Evolução } & \multirow{3}{*}{ OR } & \multirow{3}{*}{ IC (95\%) } & \multirow{3}{*}{$\mathrm{p}$-valor } \\
\hline & \multicolumn{2}{|c|}{ Óbito } & \multicolumn{2}{|c|}{ Alta } & & & \\
\hline & $\mathrm{N}$ & $\%$ & $\mathrm{~N}$ & $\%$ & & & \\
\hline \multicolumn{8}{|l|}{ Sexo } \\
\hline Masculino & 52 & 44,1 & 66 & 55,9 & 1,0 & - & - \\
\hline Feminino & 35 & 43,2 & 46 & 56,8 & 0,96 & $0,54-1,70$ & 0,9046 \\
\hline Total & 87 & 43,7 & 112 & 56,3 & - & - & - \\
\hline \multicolumn{8}{|l|}{ Faixa etária (anos) $^{\star}$} \\
\hline Menor de 65 & 28 & 44,4 & 35 & 55,6 & 1,0 & - & - \\
\hline Menor ou igual a 65 & 59 & 43,7 & 76 & 56,3 & 0,97 & $0,53-1,77$ & 0,922 \\
\hline Total & 87 & 43,9 & 111 & 56,1 & - & - & - \\
\hline
\end{tabular}

* 1 paciente não referiu a idade

Tabela 2 - Associação das Variáveis: Tempo de Internação, Existência de Doença Crônica e Diagnóstico de Admissão segundo a Evolução dos Pacientes Admitidos na UTI.

\begin{tabular}{|c|c|c|c|c|c|c|c|}
\hline \multirow{3}{*}{ Variáveis } & \multicolumn{4}{|c|}{ Evolução } & \multirow{3}{*}{ OR } & \multirow{3}{*}{ IC (95\%) } & \multirow{3}{*}{ p-valor } \\
\hline & \multicolumn{2}{|c|}{ Óbito } & \multicolumn{2}{|c|}{ Alta } & & & \\
\hline & $\mathrm{N}$ & $\%$ & $\mathrm{~N}$ & $\%$ & & & \\
\hline \multicolumn{8}{|l|}{ Tempo de internação* } \\
\hline Menos de $72 \mathrm{~h}$ & 46 & 37,7 & 76 & 62,3 & 1,0 & - & \\
\hline Mais de $72 \mathrm{~h}$ & 40 & 53,3 & 35 & 46,7 & 1,88 & $1,05-3,38$ & 0,032 \\
\hline Total & 86 & 46,2 & 111 & 53,8 & - & - & - \\
\hline \multicolumn{8}{|l|}{ Existência de alguma doença crônica** } \\
\hline Não & 14 & 30,4 & 32 & 69,6 & 1,0 & - & - \\
\hline Sim & 72 & 47,4 & 80 & 52,6 & 2,05 & $1,01-4,15$ & 0,0448 \\
\hline Total & 86 & 39,3 & 112 & 60,7 & - & - & - \\
\hline Diagnóstico da admissão da UTI*** & & & & & & & 0,4168 \\
\hline Cirurgia eletiva ou de urgência & 07 & 31,8 & 15 & 68,2 & 1,0 & - & - \\
\hline Politraumatismo & 02 & 28,6 & 05 & 71,4 & 0,85 & $0,13-5,55$ & $1,000^{\star \star \star \star}$ \\
\hline Doença clínica aguda & 73 & 44,2 & 92 & 55,8 & 1,69 & $0,65-4,38$ & 0,2728 \\
\hline Total & 82 & & 112 & & & & \\
\hline
\end{tabular}

* 2 pacientes sem informação do tempo de internação,

** 1 paciente sem informação da existência de doença crônica

*** 5 pacientes sem definição do diagnóstico de admissão

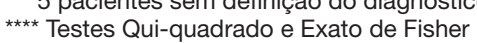


de 2,76; 4,43 e 3,06, respectivamente. O lactato $>4$ $\mathrm{mmol} / \mathrm{L}$ foi fator de risco para óbito com OR de 5,86.

Tanto o escore SOFA quanto o APACHE II estiveram associados com a evolução, sendo ambos fatores de risco para o óbito, com OR iguais a 3,92 e 8,74, respectivamente, quando o APACHE foi superior a $25 \mathrm{e}$ o SOFA a 12 pontos. Em relação aos órgãos acometidos, ter mais de três órgãos com disfunção (conforme avaliado pelo SOFA) esteve relacionado com risco três vezes maior de óbito (Tabelas 4 e 5).

Após o ajuste das variáveis com a regressão logística, apenas o tempo prévio de internação anterior à chegada à UTI maior do que 72 horas, a presença de doença crônica associada, o número de órgãos acometidos superior a três e o lactato maior que $4 \mathrm{mmol} / \mathrm{L}$ estiveram associados com o óbito (Tabela 6).

Tabela 3 - Associação das Variáveis de Tempo para o Diagnóstico de Sepse, Órgão de Origem da Sepse e Isolamento de Agente Etiológico com a Evolução dos Pacientes Admitidos na UTI.

\begin{tabular}{|c|c|c|c|c|c|c|c|}
\hline \multirow{3}{*}{ Variáveis } & \multicolumn{4}{|c|}{ Evolução } & \multirow{3}{*}{ OR } & \multirow{3}{*}{ IC (95\%) } & \multirow{3}{*}{$\mathrm{p}$-valor } \\
\hline & \multicolumn{2}{|c|}{ Óbito } & \multicolumn{2}{|c|}{ Alta } & & & \\
\hline & $\mathrm{N}$ & $\%$ & $\mathrm{~N}$ & $\%$ & & & \\
\hline \multicolumn{8}{|l|}{ Sepse $^{\star}$} \\
\hline Na admissão na UTI & 65 & 44,8 & 80 & 55,2 & 1,0 & - & \\
\hline Após admissão na UTI & 10 & 41,7 & 14 & 58,3 & 0,87 & $0,36-2,10$ & 0,773 \\
\hline Total & 75 & 44,4 & 94 & 55,6 & - & - & - \\
\hline \multicolumn{8}{|l|}{ Sepse pulmonar** } \\
\hline Sim & 58 & 43,3 & 76 & 56,7 & 1,0 & - & \\
\hline Não & 16 & 47,1 & 18 & 52,9 & 1,16 & $0,54-2,47$ & 0,692 \\
\hline Total & 74 & 44,0 & 94 & 55,0 & - & - & - \\
\hline \multicolumn{8}{|l|}{ Sepse urinária** } \\
\hline Sim & 09 & 39,1 & 14 & 60,9 & 1,0 & - & \\
\hline Não & 65 & 44,8 & 80 & 51,2 & 1,26 & $0,51-3,10$ & 0,609 \\
\hline Total & 74 & 44,0 & 94 & 55,0 & - & - & - \\
\hline \multicolumn{8}{|l|}{ Sepse (número de órgãos)** } \\
\hline 1 & 15 & 45,5 & 18 & 54,5 & 1,0 & - & \\
\hline 2 e mais & 59 & 43,7 & 76 & 56,3 & 0,93 & $0,43-2,00$ & 0,856 \\
\hline Total & 74 & 44,0 & 94 & 55,0 & - & - & - \\
\hline \multicolumn{8}{|l|}{ Agente etiológico isolado*** } \\
\hline Sim & 27 & 40,3 & 40 & 59,7 & 1,0 & - & \\
\hline Não & 46 & 46,0 & 54 & 54,0 & 1,26 & $0,67-2,36$ & 0,467 \\
\hline
\end{tabular}

*30 pacientes sem informação sobre intervalo de tempo para início da sepse

**31 pacientes sem informação do órgão de origem da sepse

***32 pacientes sem informação sobre isolamento do agente etiológico.

Tabela 4 - Associação das Variáveis Clínicas segundo a Evolução dos Pacientes Admitidos na UTI, no Momento de Diagnóstico de Sepse.

\begin{tabular}{|c|c|c|c|c|c|c|c|}
\hline \multirow{3}{*}{ Variáveis Clínicas } & \multicolumn{4}{|c|}{ Evolução } & \multirow{3}{*}{ OR } & \multirow{3}{*}{ IC (95\%) } & \multirow{3}{*}{$\mathrm{p}$-valor } \\
\hline & \multicolumn{2}{|c|}{ Óbito } & \multicolumn{2}{|c|}{ Alta } & & & \\
\hline & $\mathrm{N}$ & $\%$ & $\mathrm{~N}$ & $\%$ & & & \\
\hline \multicolumn{8}{|c|}{ Pressão arterial média $(\mathrm{mmHg})^{*}$} \\
\hline Maior que 70 & 48 & 37,5 & 80 & 62,5 & 1,0 & - & \\
\hline Menor ou igual a 70 & 31 & 50,8 & 30 & 49,2 & 1,72 & $0,93-3,19$ & 0,083 \\
\hline Total & 79 & & 110 & & & & \\
\hline \multicolumn{8}{|c|}{ Número de órgãos acometidos ${ }^{\star *}$} \\
\hline De 1 a 3 & 31 & 30,7 & 70 & 69,3 & 1,0 & - & \\
\hline De 4 a 6 & 48 & 57,1 & 36 & 42,9 & 3,01 & $1,64-5,51$ & 0,001 \\
\hline Total & 79 & & 106 & & & & \\
\hline
\end{tabular}

*10 pacientes sem informação sobre pressão arterial média (pressão sistólica menos a pressão diastólica, dividido por 3 e somado a pressão diastólica).

**14 pacientes em informação sobre disfunção de órgãos 
A associação do escore SOFA com os níveis de AT e plaquetas mostrou-se significativa, pois tanto a atividade da AT $<70 \%$ quanto as plaquetas $<100.000 / \mathrm{mm}^{3}$ estiveram relacionadas com o SOFA $\geq 12$ pontos (Tabela 7). O esco- re SOFA mostrou associação com o intervalo de tempo para o óbito, assim, um terço dos pacientes com pontuação superior a 12 faleceram em menos de $72 h(p=0,006$, $\mathrm{OR}=7,08$, IC 95\% = 1,48- 37,5) (Tabela 8).

Tabela 5 - Associação dos Escores APACHE II e SOFA com a Evolução dos Pacientes Admitidos na UTI.

\begin{tabular}{|c|c|c|c|c|c|c|c|}
\hline \multirow{3}{*}{ Variáveis } & \multicolumn{4}{|c|}{ Evolução } & \multirow{3}{*}{ OR } & \multirow{3}{*}{ IC (95\%) } & \multirow{3}{*}{$\mathrm{p}$-valor } \\
\hline & \multicolumn{2}{|c|}{ Óbito } & \multicolumn{2}{|c|}{ Alta } & & & \\
\hline & $\mathrm{N}$ & $\%$ & $\mathrm{~N}$ & $\%$ & & & \\
\hline \multicolumn{8}{|l|}{ Escore APACHE II } \\
\hline Menor que 25 & 62 & 38,8 & 98 & 61,2 & 1,0 & - & \\
\hline Maior ou igual a 25 & 20 & 71,4 & 08 & 29,6 & 3,92 & $1,64-9,52$ & 0,003 \\
\hline Total $^{\star}$ & 82 & & 106 & & & & \\
\hline \multicolumn{8}{|l|}{ SOFA } \\
\hline Menor que 12 & 49 & 32,9 & 100 & 67,1 & 1,0 & - & \\
\hline Maior ou igual a 12 & 30 & 81,1 & 07 & 18,9 & 8,74 & $3,58-21,3$ & 0,000 \\
\hline Total $^{\star \star}$ & 79 & & 107 & & & & \\
\hline
\end{tabular}

* 11 pacientes sem informação do APACHE, ** 13 sem informação do SOFA.

Tabela 6 - Modelo Final da Análise Multivariada após Seleção por Blocos. Tempo de Internação, Doença Crônica, Órgãos Acometidos, INR e Lactato.

\begin{tabular}{|c|c|c|c|}
\hline Fatores de risco & OR & $\mathrm{Cl} 95 \%$ & $\mathrm{p}$-valor \\
\hline \multicolumn{4}{|l|}{ Tempo de internação } \\
\hline Menos de $72 \mathrm{~h}$ & 1,00 & - & - \\
\hline Mais de $72 \mathrm{~h}$ & 2,05 & $1,02-4,11$ & 0,0426 \\
\hline \multicolumn{4}{|l|}{ Doença crônica } \\
\hline Não & 1,00 & - & - \\
\hline Sim & 4,69 & $1,57-13,9$ & 0,0055 \\
\hline \multicolumn{4}{|c|}{ Número de órgãos acometidos } \\
\hline De 1 a 3 & 1,00 & - & - \\
\hline De 4 a 6 & 3,01 & $1,51-5,99$ & 0,0017 \\
\hline INR & & & 0,0629 \\
\hline Menor ou igual a 1,5 & 1,00 & - & - \\
\hline Maior que 1,5 & 1,95 & $0,91-4,19$ & 0,0854 \\
\hline Não verificada & 3,31 & $1,05-10,4$ & 0,0404 \\
\hline Lactato & & & 0,0240 \\
\hline Menor ou igual a 4 & 1,00 & - & - \\
\hline Maior de 4 & 5,05 & $1,45-17,6$ & 0,0110 \\
\hline Não verificado & 1,97 & $0,94-4,13$ & 0,0711 \\
\hline
\end{tabular}

Saiu do modelo a contagem de plaquetas

Tabela 7 - Associação das Variáveis Antitrombina e Plaquetas com o Escore SOFA dos Pacientes Admitidos na UTI.

\begin{tabular}{|c|c|c|c|c|c|}
\hline \multirow{3}{*}{ Variáveis } & \multicolumn{4}{|c|}{ SOFA } & \multirow{3}{*}{$\mathrm{p}$-valor } \\
\hline & \multicolumn{2}{|c|}{$<12$} & \multicolumn{2}{|c|}{$\geq 12$} & \\
\hline & $\mathrm{N}$ & $\%$ & $\mathrm{~N}$ & $\%$ & \\
\hline \multicolumn{6}{|l|}{ Antitrombina III* } \\
\hline Menor ou igual a 70 & 13 & 50,0 & 13 & 50,0 & 0,002 \\
\hline \multicolumn{6}{|l|}{ Plaquetas ${ }^{\star \star}$} \\
\hline
\end{tabular}

* 3 pacientes tinham informação sobre o valor da antitrombina, porém não tinham o SOFA completo

**7 pacientes tinham informação sobre a contagem das plaquetas, porém não tinham o SOFA completo 
Tabela 8 - Associação das Variáveis Antitrombina, Plaquetas, os Escores SOFA e APACHE II com Intervalo de Tempo para o Óbito

\begin{tabular}{|c|c|c|c|c|c|}
\hline \multirow{3}{*}{ Variáveis } & \multicolumn{4}{|c|}{ Intervalo para Óbito } & \multirow{3}{*}{ p-valo } \\
\hline & \multicolumn{2}{|c|}{$\leq 72 \mathrm{hs}$} & \multicolumn{2}{|c|}{$>72 \mathrm{hs}$} & \\
\hline & $\mathrm{N}$ & $\%$ & $\mathrm{~N}$ & $\%$ & \\
\hline \multicolumn{6}{|l|}{ Antitrombina III } \\
\hline Maior que 70 & 01 & 5,0 & 19 & 95,0 & \\
\hline Menor ou igual a 70 & 02 & 11,8 & 15 & 88,2 & $0,584^{\star \star}$ \\
\hline \multicolumn{6}{|l|}{ Plaquetas $^{*}$} \\
\hline Maior que 100.000 & 06 & 13,6 & 38 & 86,4 & \\
\hline Menor ou igual a 100.000 & 05 & 21,7 & 18 & 78,3 & $0,491^{\star \star}$ \\
\hline \multicolumn{6}{|l|}{ Escore APACHE } \\
\hline Menor que 25 & 06 & 10,5 & 51 & 89,5 & \\
\hline Maior ou igual a 25 & 05 & 26,3 & 14 & 73,7 & $0,129^{\star \star}$ \\
\hline \multicolumn{6}{|l|}{ SOFA $^{\star * \star}$} \\
\hline Menor que 12 & 03 & 6,7 & 42 & 93,3 & \\
\hline Maior ou igual a 12 & 09 & 33,3 & 18 & 66,7 & $0,006^{\star \star}$ \\
\hline
\end{tabular}

* 01 paciente tinha contagem de plaquetas, porém não tinha intervalo de tempo para óbito.

** Testes Qui-quadrado e Exato de Fisher.

*** SOFA: OR = 7,08, IC 95\% (1,48-37-5).

\section{DISCUSSÃO}

Neste estudo, após a regressão logística, nenhum exame de coagulação se mostrou bom indicador prognóstico de óbito de maneira isolada; o mesmo foi observado por Pettila e col. avaliando o poder discriminatório da atividade da AT quanto à associação com a mortalida$\mathrm{de}^{23}$. Alguns autores, porém, quando analisaram de forma univariada observaram que a baixa atividade da AT, a plaquetopenia e o alargamento do INR representaram um aumento no risco de morte, fato esse, que condiz com os resultados aqui descritos 8 ,19,24-29.

Knaus e col. observaram que os pacientes que foram admitidos na UTI provenientes da emergência tinham menor mortalidade do que os pacientes que estavam internados há mais tempo, do mesmo modo que os pacientes que tinham sepse urinária ${ }^{13}$. Neste estudo, o órgão de origem da sepse não influenciou o prognóstico, porém, o tempo de internação hospitalar anterior à admissão na UTI esteve associada com maior mortalidade.

Brun-Buisson e col. não evidenciaram associação entre o prognóstico e a positividade ou negatividade das culturas, fato esse também apreciado no presente estudo, o que sugere que o prognóstico está mais relacionado à gravidade da sepse do que da infecção subjacente ${ }^{30}$.

As comorbidades estiveram associadas a maior risco de mortalidade, e isso pode ser devido a um efeito aditivo negativo do distúrbio fisiológico agudo em paciente cronicamente enfermo. Foreman e col. descreveram que os pacientes cirróticos têm maior risco de desenvolver sepse e de morrer dela ${ }^{31}$. Alberti e col. observaram que comorbidades como a doença hepática crônica, insufi- ciência cardíaca e imunossupressão estavam associadas com pior prognóstico ${ }^{32}$. De forma geral, a disfunção de órgãos aguda está relacionada com o prognóstico em curto prazo, e as comorbidades em longo prazo ${ }^{3}$.

As concentrações de lactato podem ser usadas para avaliar a gravidade da doença na UTI, pois estiveram associadas com a mortalidade nas análises uni e multivariada. Foi evidenciado por Nguyen e col. que a redução na concentração do lactato nas primeiras $6 \mathrm{~h}$ de tratamento intensivo estava associada, de forma independente, com redução na mortalidade ${ }^{33}$. A hipóxia tecidual indicativa de doença grave, é preditiva do desenvolvimento de $\mathrm{DMOS}^{34}$. O lactato, como é um indicador de hipóxia e hipoperfusão tecidual, está intimamente relacionado com a gravidade dos pacientes sépticos, e com maior risco de óbito ${ }^{35}$.

A disfunção de órgãos, avaliada pelo SOFA, foi bom preditor de mortalidade geral e também esteve associado a maior mortalidade precoce na amostra estudada. Garnancho-Montero e col. descreveram que o escore SOFA da admissão, assim como o aumento do SOFA nos três primeiros dias de admissão na UTI estiveram associados com maior risco de morte ${ }^{36}$.

Com relação a DMOS, foi possível observar que tanto a baixa atividade da AT, como a plaquetopenia, aumentou o risco de disfunção orgânica, fato esse também ob-

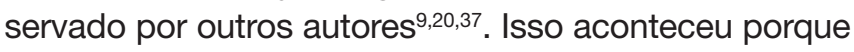
a hipóxia tecidual e o processo inflamatório da sepse podem ativar direta ou indiretamente a cascata da coagulação, o que ocasionou a formação de trombos na micro-circulação, que agrava ainda mais a perfusão tecidual resultando em disfunção aguda de órgãos ${ }^{37}$. 
Ueno e col. associaram a plaquetopenia e a DMOS e concluíram que a lesão endotelial vascular foi a causa primária de DMOS em pacientes com plaquetopenia ${ }^{37}$. Okabayashi e col. descreveram que o SOFA estava aumentado nos pacientes com coagulopatia e que os parâmetros hemostáticos e, principalmente a atividade da AT, é indicador útil na falência de órgãos ${ }^{20}$.

\section{CONCLUSÃO}

O lactato sérico elevado, o tempo de hospitalização maior que $72 \mathrm{~h}$ prévio a transferência para a UTI, e a presença de comorbidades estão associados com maior risco de morte. Pontuação elevada nos escore SOFA e APACHE II também apresentam a mesma associação, confirmando a universalidade destes escores para predizer mortalidade. A disfunção de órgãos está associada com alterações da coagulação como plaquetopenia e baixa atividade da AT.

O presente estudo confirmou que os exames laboratoriais, particularmente os que refletem aspectos da coagulação, não podem predizer o risco de morte isoladamente, o que pode ser mais bem avaliado pelos escores SOFA e APACHE II. Entre os exames, apenas o lactato sérico, quando elevado, esteve relacionado com maior risco de óbito.

\section{REFERÊNCIAS}

01. Sibbald WJ, Neviere R - Pathophysiology of sepsis. 2005 Up To Date, www.uptodate.com [Acessado Abril 2005]

02. Bilevicius E, Dragosavac D, Dragosavac $S$ et al - Multiple organ failure in septic patients. Braz J Infect Dis, 2001;5:103-110.

03. Silva E, Pedro Mde A, Sogayar AC et al - Brazilian Sepsis Epidemiological Study (BASES study). Crit Care, 2004:8:R251-R260.

04. Angus DC, Linde-Zwirble WT, Lidicker $\mathrm{J}$ et al - Epidemiology of severe sepsis in the United States: analysis of incidence, outcome, and associated costs of care. Crit Care Med, 2001;29:1303-1310.

05. Finfer S, Bellomo R, Lipman J et al - Adult-population incidence of severe sepsis in Australian and New Zealand intensive care units. Intensive Care Med, 2004;30:589-596.

06. Takala A, Nupponen I, Kylanpaa-Back ML et al - Markers of inflammation in sepsis. Ann Med, 2002;34:614-623.

07. Bernard GR, Vincent JL, Laterre PF et al - Efficacy and safety of recombinant human activated protein $\mathrm{C}$ for severe sepsis. N Engl J Med, 2001;344:699-709

08. Yan SB, Helterbrand JD, Hartman DL et al - Low levels of protein C are associated with poor outcome in severe sepsis. Chest, 2001;120:915-922.

09. Kidokoro A, Iba T, Fukunaga $\mathrm{M}$ et al - Alterations in coagulation and fibrinolysis during sepsis. Shock, 1996:5:223-228.

10. Vervloet MG, Thijs LG, Hack CE - Derangements of coagulation and fibrinolysis in critically ill patients with sepsis and septic shock. Semin Thromb Hemost, 1998;24:33-44

11. Martin GS, Mannino DM, Eaton $S$ et al - The epidemiology of sepsis in the United States from 1979 through 2000. N Engl J Med, 2003;348:1546-1554.

12. Marshall JC, Vincent JL, Fink MP - Measures, markers, and mediators: toward a staging system for clinical sepsis. A report of the Fifth Toronto Sepsis. Roundtable, Toronto, Ontario, Canada, Oct 25-26, 2000. Crit Care Med, 2003;31:1560-1567.

13. Knaus WA, Draper EA, Wagner DP et al - APACHE II: a severity of disease classification system. Crit Care Med, 1985;13:818-829.

14. Vincent JL, Moreno R, Takala J et al - The SOFA (Sepsis-related Organ Failure Assessment) score to describe organ dysfunction/failure. Intensive Care Med, 1996;22:707-710.

15. Shorr AF, Thomas SJ, Alkins SA et al - D-dimer correlates with proinflammatory cytokine levels and outcomes in critically ill patients. Chest, 2002:121:1262-1268.

16. Stephan F, Montblanc JD, Cheffi A et al - Thrombocytopenia in critically ill surgical patients: a case-control study evaluating attributable mortality and transfusion requirements. Crit Care, 1993;3:151-158.

17. Vincent JL, Yaguchi A, Pradier O - Platelet function in sepsis. Crit Care Med, 2002;30:(Suppl5):S313-S317.

18. Wada $\mathrm{H}$, Mori $\mathrm{Y}$, Okabayashi $\mathrm{K}$ et al - High plasma fibrinogen level is associated with poor clinical outcome in DIC patients. Am J Hematol, 2003;72:1-7.

19. Wilson RF, Mammen EF, Tyburski JG et al - Antithrombin levels related to infections and outcome. J. Trauma, 1996;40:384-387.

20. Okabayashi $\mathrm{K}$, Wada $\mathrm{H}$, Ohta $\mathrm{S}$ et al - Hemostatic markers and the sepsis-related organ failure assessment score in patients with disseminated intravascular coagulation in an intensive care unit. Am J Hematol, 2004;76:225-229.

21. American College of Chest Physicians and Society of Critical Care Medicine Consensus Conference: definitions for sepsis and organ failure and guidelines for the use of innovative therapies in sepsis. Crit Care Med, 1992;20:864-874

22. Koury JCA, Lacerda HR, Barros Neto AJ - Características da população com sepse em unidade de terapia intensiva de hospital terciário e privado da cidade do Recife. RBTI, 2006; 18:52-58.

23. Pettila V, Pentti J, Pettila M et al - Predictive value of antithrombin III and serum C-reactive protein concentration in critically ill patients with suspected sepsis. Crit Care Med, 2002;30:271-275.

24. Fourrier F, Chopin C, Goudemand S et al - Septic shock, multiple organ failure, and disseminated intravascular coagulation. Compared patterns of antithrombin III, protein C, and protein S deficiencies. Chest, 1992;101:816-823

25. Gando S, Kameue T, Nanzaki S et al - Disseminated intravascular coagulation is a frequent complication of systemic inflammatory response syndrome Thromb Haemost, 1996;75:224-228.

26. Mesters RM, Mannucci PM, Coppola R et al - Factor VII and antithrombin III activity during severe sepsis and septic shock in neutropenic patients. Blood, 1996:88:881-886

27. Martinez MA, Pena JM, Fernandez A et al - Time course and prognostic significance of hemostatic changes in sepsis: relation to tumor necrosis factor-alpha. Crit Care Med, 1999;27:1303-1308.

28. Hesselvik JF, Blomback M, Brodin B et al - Coagulation fibrinolysis, and kallikrein systems in sepsis: relation to outcome. Crit Care Med, 1989;17:724-733.

29. Lorente JA, Garcia-Frade LJ, Landin L et al - Time course of hemostatic abnormalities in sepsis and its relation to outcome. Chest, 1993;103:1536-1542.

30. Brun-Buisson C, Doyon F, Carlet J - Bacteremia and severe sepsis in adults: a multicenter prospective survey in ICUs and wards of 24 hospitals. Am J Respir Crit Care Med, 1996;154:617-624.

31. Foreman MG, Mannino DM, Moss M - Cirrhosis as a risk factor for sepsis and death: analysis of a National Hospital Discharge Survey. Chest, 2003;124:1016-1020.

32. Alberti C, Brun-Buisson C, Goodman SV et al - Influence of systemic inflammatory response syndrome and sepsis on outcome of critically ill Infected patients. Am J Respir Crit Care Med, 2003;168:77-84.

33. Nguyen HB, Rivers EP, Knoblich BP - Early lactate clearance is associated with improved outcome in severe sepsis and septic shock. Crit Care Med, 2004;32:1637-1642.

34. Rivers E, Nguyen B, Havstad S et al - Early goal-therapy in the treatment of severe sepsis and septic shock. N Engl J Med, 2001;345:1368-1377.

35. Silva E- Coagulopatia na Sepse, em: David CM - Medicina Intensiva. Rio de Janeiro, Revinter, 2004;51:581-590.

36. Garnancho-Montero J, Garcia-Garmendia JL, Barrero-Almodovar A - Impact of adequate empirical antibiotics therapy on the outcome of patients admitted to the intensive care unit with sepsis. Crit Care Med, 2003:31:2742-2751.

37. Ueno $\mathrm{H}$, Hirasawa $\mathrm{H}$, Oda $\mathrm{S}$ et al - Coagulation/fibrinolysis abnormality and vascular endothelial damage in the pathogenesis of thrombocytopenic multiple organ failure. Crit Care Med, 2002;30:2242-2248. 